\title{
Percepções de Competência e Desenvolvimento Motor de meninos e meninas: um estudo transversal
}

Nadia Cristina Valentini*

\section{Resumo}

Os objetivos deste estudo foram investigar as percepções de competência e desempenho motor de crianças, e as relações entre estas percepções e o desempenho motor de meninos e meninas de diferentes idades. Oitenta e oito crianças foram testadas através Test of Gross Motor Development e responderam a Pictorial Scale of Perceived Competence and Social Acceptance. Os resultados sugerem que: (1) meninos e meninas de diferentes idades evidenciam percepções similares, porém não são precisos com relação a estas percepções (2) crianças mais velhas exibem desempenho locomotor superior, porém não existem diferenças nas habilidades de controle de objetos nos grupos etários; (3) meninos e meninas evidenciam desempenhos locomotores similares, porém meninos evidenciam superioridade nas habilidade de controle de objetos; (4) desenvolvimento motor atual não se configura como preditor de percepção de competência. Instrução e oportunidades de movimento são críticos para o desenvolvimento motor e para a construção de percepções de competência realísticas em crianças.

Descritores: percepções de competência, desenvolvimento motor, gênero

\footnotetext{
Abstract

The objectives ofthis study were to (1) determine gender and age differences for perceived physical competence and motor performance, and (2) exam the relationship between perceived physical competence and actual motor skill competence in children. Children $(N=88)$
}

individually completed the Harter andPike (1984) Pictorial Scale of Perceived Competence and Social Acceptance. The subject's actual motor skill performance was measured by using Ulrich 's (1985) Test ofGross Motor Development. The results suggested that: (1) boys and girls at different age group showed similar perceptions of competence, however were not precise about these perceptions; (2) older children showed a better locomotor performance, but a similar object control performance when compared to younger children; (3) boys and girls had a similar locomotor performance, however boys were superior on the object control performance when compared to girls; (4) actual motor performance is not apredictor of perceived competence. Movement instruction and opportunities are criticai for motor development and realistic perceptions of competence.

\section{Introdução}

Percepções de competência, ou os julgamentos expressados pelo indivíduo sobre suas habilidades em diferentes domínios têm sido o interesse de vários pesquisadores nas últimas duas décadas. Este interesse investigativo não cresceu artificialmente, e sim da inflexão da prática que nos desafia a entender o complexo e dinâmico processo da motivação humana. Como as percepções de competência são construídas, os fatores que as influenciam (e são influenciados por ela), bem como a sua expressão, parecem críticos componentes da motivação para a aprendizagem. Os padrões de motivação adotados por crianças na participação esportiva ou em outros contextos de conquistas pessoais e aprendizagens, parecem ser fortemente influenciados pelas percepções de competência que estas crianças evidenciam. Muitos dos estudos voltados à investi- 
Os padrōes de $\mid$ gação da motivação motivação adotados infantil têm como por crianças na referencial teórico o

participação modelo de percepção esportiva ou em de competência prooutros contextos de posto por Harter conquistas pessoais e (1978). Esta pesquisaaprendizagens, dora propõe o entenparecem ser fortemente influenciados pelas percepções de competência que estas crianças evidenciam. dimento das percepções de competência a partir de um modelo multidimensional, onde a competência infantil é demonstrada nos domínios

ognitivo, social (ou interpessoal) e físico. A expressão de diferentes percepções de competências em domínios específicos parece ser construída muito cedo na infância. Estudos evidenciam que crianças partir de 4 anos expressam percepções de competências relacionadas a domínios específicos (HARTER, 1978,1982,1984).

No contexto de aprendizagem crianças que percebem a si mesmas como altamente competentes em uma atividade ou habilidade tendem a persistir por mais tempo e continuar em tentativas de executar a habilidade com maestria, mesmo quando encontram dificuldades. Ao contrário, crianças que percebem a si próprias como pouco competentes tendem a desistirem ou perderem $o$ interesse na maestria de habilidades ou tarefas, principalmente quando encontram dificuldades (FELTZ, 1983; HARTER,1978; HARTER, 1992; KLINT, 1987; RUDISILL, 1989; VALENTINI, 1999).

Nesta perspectiva motivacional, como a percepção de competência é construída e sua precisão são fatores críticos no processo de desenvolvimento infantil. Harter (1978) sugere que a motivação para a competência na criança é estruturada sobre quatro fatores, os quais parecem influenciar níveis mais elevados ou mais baixos de percepção de competência. Estes fatores incluem: (a) experiências passadas; (b) dificuldades ou desafios associados com o resultado da tarefa; (c) suporte e interação pessoal com outros que são significativos para a criança; e (d) motivação intrínseca. Uma criança que exibe pouca motivação intrínseca talvez esteja menos consciente de sua competência por possuir poucas experiências prévias, conhecimento limitado dos desafios da tarefa proposta e, no decorrer das experiências, por receber pouco suporte de pessoas significativas (pais, professores, pares, entre outros).

Crianças que não evidenciam conscientização de sua competência atual são mais vulneráveis no sentido de superestimar ou subestimar sua competência atual (ULRICH, 1978). Estas duas formas de julgamento influenciam decisivamente a aprendizagem da criança. $\mathrm{Ou}$ seja, superestimar a competência talvez leve a criança a expectativas não realistas no desempenho de tarefas e fracassos no processo e resultados. Por exemplo, experienciar fracassos quando a tarefa não é percebida como difícil talvez resulte em baixa percepção de competência (HARTER, 1982). Subestimar competência conduz a elaboração de baixas expectativas por parte da criança em relação a sua competência futura, o que influencia conseqüentemente desempenho e motivação para persistir.

A precisão com que a criança percebe sua própria competência é, portanto, fundamental no estudo da motivação para a aprendizagem de diferentes tarefas, incluindo as motoras. A discrepância entre percepções de competência física expressada pela criança e seu desenvolvimento motor atual talvez influencie negativamente a motivação da criança para conquistar ou persistir nas atividades fisicas (GOODWAY, 1997; STTPEK, 1990; RUDISILL, 1993;ULRICH, 1987, WEISS, 1990). A inves-tigação dos fatores que influenciam esta precisão ou discrepância entre a percepção e a competência real atual, ainda têm sido pouco discutidas na literatura.

$\mathrm{O}$ fator idade tem sido apontado em alguns estudos como influente na discrepância entre as percepções de competência e desenvolvimento motor atual. Estudos evidenciam que crianças entre 5 e 11 anos de idade avaliam seus desempenhos motores, porém, as mais jovens não são muito precisas sobre estas percepções (HARTER,1982;

HARTER,1984;RUDISILL,1993;URICH， 1987). Crianças mais jovens, por volta de 5 anos de idade, freqüentemente reportam percepções de competência física elevada embora demonstrando desempe- 
nho motor real baixo(GOODWAY-SHEBLER, 1994; OVERBY， 1994;ULRICH， 1987).Em geral,observa-se que as percepções de competência de crianças são inicialmente altas, permanecendo relativamente altas e constantes por um período de tempo, paralelo ao qual ocorre o enriquecimento das habilidades motoras, resultando, com o passar do tempo e com o desenvolvimento da criança, em percepções mais precisas e realistas da competência fisica real(RUDISILL, 1993;ULRICH, 1987).

Em um estudo transversal, Rudisill (1993) investigou as relações entre percepção de competência e desenvolvimento motor atual de crianças com desenvolvimento típico entre 9 e 11 anos. As análises de regressão deste estudo revelaram que as relações entre desenvolvimento motor real e percepções de competência eram moderadamente correlacionadas. $\mathrm{O}$ fato de adicionar idade no modelo de regressão múltipla levou ao fortalecimento da correlação, enquanto que incluindo gênero no modelo não foram observadas alterações. Crianças mais velhas evidenciaram níveis mais altos de desenvolvimento motor que as crianças mais jovens, entretanto, não reportaram alterações nas percepções de competência. Em outras palavras, com o aumento da idade, o desenvolvimento motor melhorou e as percepções de competência permaneceram as mesmas.

Os resultados de Rudisill (1993) são similares aos resultados prévios de Harter (1981) eUlrich (1987) os quais sugerem a observação de um possível platô por volta dos 8 anos até os 12 anos de idade na precisão das percepções em níveis moderados. Entretanto, considerando a perspectiva teórica proposta por Harter (1985) questionamentos podem ser levantados em relação a precisão na percepção e auto-avaliação de competência de crianças que em geral possuem poucas oportunidades de prática motora. Goodway e Rudisill (1997), investigando crianças que demonstravam atrasos no desenvolvimento motor, sugerem que estas crianças não são precisas quanto suas competências motoras reais. Estas autoras sugerem que é necessário propiciar instrução e oportunidades apropriadas de prática de movimentos para desenvolver percepções pessoais precisas sobre competências.

Em relação às percepções de competência física e gê- nero, vários estudos sugerem a ausência de diferenças nas percepções de meninos e meninas (ANDERSON, 1985; GOODWAY, 1996,1997;HANSFORD, 1982; VAEENTINI, 1999; WYLIE, 1979).Outros estudos,en-tretanto, sugerem que meninos tendem a reportar índices mais elevados nas percepções de competência física quando comparado com meninas (FELTZ, 1984; RUDISILL, 1993;ULRICH, 1987).

Portanto, percepção de competência é considerada um mediador importante na manutenção ou aumento da motivação, especialmente de crianças, pois a forma como a criança percebe sua competência, influencia suas razões para conquistar e persistir em atividades de movimento. Nos parece, portanto, fundamental ao desenvolvimento da criança propiciar oportunidades para a construção de percepções elevadas de competência através do desenvolvimento da competência motora real da criança em diferentes habilidades fundamentais.

O desenvolvimento de habilidades motoras fundamentais, na perspectiva de uma prática apropriada ao nível de desenvolvimento infantil, deveria se constituir em foco de atenção e prioridade de programas de movimento. As habilidades motoras fundamentais são consideradas "blocos de construção" indispensáveis para o desenvolvimento de atividades de movimento em uma perspectiva de vida ativa e saudável, bem como para a especialização de habilidades motoras específicas da dança e/ou esportes (GABARD, 2000; HAYWOOD,2001;PAYNE, 1999;SEEFELDT, 1982).É importante ressaltar que estas habilidades não "emergem naturalmente" (NEWELL, 1984) durante a infância, elas são resultados de vários fatores que em constante interação, influenciam o desenvolvimento motor da criança, entre eles o contexto de ensino, a motivação, o desenvolvimento neurológico, as condições sociais e culturais e as experiências passadas (GABBARD, 2000;HAYWOOD,2001;NEWELL 1984).

Crianças devem aprender um certo nível de competência nas habilidades motoras fundamentais para engajarem-se com sucesso (sucesso entendido neste contexto como participação efetiva na atividade), em habilidades esportivas específicas que contribuam 
A participação futura para uma melhor qualiem esportes e dade de vida atividades de (SEEFELDT, 1980; movimento que SEEFELDT,1982).Na enriqueçam a perspectiva do qualidade da vida referencial teórico aqui depende de prática de adotado, a educação fímovimento em níveis sica nos primeiros anos moderados e do ensino fundamental vigorosos durante a deve construir oportuinfância, a prática nidades efetivas para o intensa só é desenvolvimento de nío bservável quando existe o domínio de habilidades motoras fundamentais. veis básicos de proficiência no desempenho das habilidades motoras fundamentais. A partici-pação futura em

es e atividades de movimento que enriqueçam a qualidade da vida depende de prática de movimento em níveis moderados e vigorosos durante a infância, a prática intensa só é observável quando existe o domínio de habilidades motoras fundamentais. Crianças e jovens que não se engajam com freqüência e de maneira vigorosa (que produza ganhos na aptidão física e ou otimização das habilidades motoras esportivas) na atividade física durante os anos escolares não incorporam a prática das mesmas na sua vida adulta.

Nas pesquisas mais recentes sobre o desenvolvimento infantil nas mais variadas habilidades motoras fundamentais, os interesses investigativos bem como os objetivos são conduzidos sobre diferentes perspectivas. Entre alguns destes objetivos, podemos citar o interesse por: (1) conhecer os diferentes perfis infantis e a variabilidade entre as crianças na progressão para habilidades motoras mais maduras (LANGERDOREEN, 2002; GARCIA, 2002); (2) entender a influência do contexto no desenvolvimento de padrões mais avançados de habilidades motoras fundamentais (SOUTHARD, 2002;HAMILTON,2002);(3)entendereintervirna estruturação de padrões mais elevados para as habilidades motoras fundamentais de crianças com atrasos no desenvolvimento(VALENTINI, 1999;GOODWAY, 2002), com desvantagens sócio-econômicas

\section{(GOODWAY,1997;HAMILTON,1999),que}

nascem e vivem em contextos considerados de alto risco $\mathrm{e}$ ou fracassos constantes (GOODWAY,1996).

O interesse no perfil motor de crianças de desenvolvimento típico está também presente na literatura. Os resultados das pesquisas brasileiras apontam para níveis elementares de desenvolvimento em diferentes habilidades motoras fundamentais. Por exemplo, alguns estudos com o padrão fundamental da corrida (FERRAZ, 1992;SURDIeKREBS, 1999,PELEGRINIeCATUZZO, 1998) evidenciam estágios elementares de desenvolvimento da corrida de crianças com idades bastante diferenciadas (entre 5 e 14 anos). Mendes e Gobbi (1991) por sua vez nos mostram poucas mudanças positivas na habilidade de receber em crianças entre 5 e 8 anos após um período de tempo, e ainda assim estas mudanças se apresentaram restritas ao gênero masculino. Surdi e Krebs (1999) investigando seis habilidades motoras fundamentais (andar sobre a trave, correr, saltar horizontalmente, arremessar, chutar e quicar) encontraram resultados em sua grande maioria em nível elementar, para pré-escolares de 6 anos de idade, sendo sugerido pelos autores a carência de oportunidades de prática diversificada e a inexistência de instrução como fatores determinantes no desempenho das crianças pesquisadas.

Possíveis diferenças entre os gêneros no desenvolvimento de habilidades motoras fundamentais também se fazem presentes em vários estudos. Em geral, esses estudos revelam que é típico para os meninos evidenciar melhor desenvolvimento motor em habilidades de controle de objetos (DINUCCI, 1976; GOODWAY, 1997;MENDES, 1991;MORRIS,1982; RUDISILL, 1993;ULRICH, 1987).

Portanto, conhecer os níveis de desenvolvimento motor de crianças é fundamental para a estruturação de programas motores que venham ao encontro das necessidades dos mais variados grupos, propiciando a elaboração de práticas mais efetivas que levem criança à construção de padrões de movimento mais avançados e que garantam a participação em atividades de movimento durante toda a vida. Somados a esta necessidade emergente, conhecer os fatores 
que influenciam o desenvolvimento das habilidades motoras em crianças se faz também prioritário. Nesta perspectiva, este estudo foi conduzido para investigar desenvolvimento motor de crianças em habilidades fundamentais e investigar um dos fatores que parece influenciar a prática, as percepções de competência que estas crianças possuem. Especificamente, os objetivos deste estudo foram investigar: (1) as percepções de competência física e níveis de desempenho motor (em habilidades de locomoção e controle de objetos) de crianças em relação as diferentes idades e ao gênero; $\mathrm{e}$ (2) as relações entre percepção de competência física e desempenho motor atual de meninos e meninas de diferentes idades.

Foram estabelecidas varias hipóteses para esta investigação: (1) crianças de diferentes idades e gênero demonstram percepções de competência física similares; (2) crianças mais velhas demonstram desempenho significantemente superior nas habilidades motoras de locomoção (correr, galopar, saltitar, salto sobre o mesmo pé, salto como os dois pés, salto com 1 pé, e corrida lateral) e nas habilidades de controle de objeto (rebater, quicar, receber, chutar, arremessar) comparadas as crianças mais jovens; (3) meninos e meninas demonstram desempenhos similares nas habilidades de locomoção; (4) meninos demonstram superioridade significativa nas habilidades de controle de objetos; (5) crianças em geral são pouco precisas na percepção de seus desempenho motores atuais; (6) a precisão com que crianças percebem seu desempenho motor atual é similar entre meninos e meninas e não evidencia aumentos com a idade, (7) desenvolvimento motor atual não se configura como um preditor da percepção de competência da criança.

\section{Participantes:}

Oitenta e oito crianças (idade entre 5 e 10 anos de idade), provenientes de cinco escolas públicas da grande Porto Alegre, foram aleatoriamente selecionadas para fazer parte deste estudo. As famílias destas crianças se distinguem por apresentarem um nível baixo de escolaridade, uma renda mensal baixa (1 a 2 salários mínimos), e residirem em casas humildes, localizadas em bairros periféricos $d$ ou pobres da cidade de Porto Alegre. Todas as crianças foram testadas através do TGMD (Test of Gross Motor Development) o qual avalia desenvolvimento motor em habilidades de locomoção e de controle de objeto. Posteriormente as crianças responderam a subescala da parte física da Pictorial Scale of Perceived Competence and Social Acceptance (PSPCSA) de Harter e Pike (1984) a qual foi utilizada com o objetivo de avaliar as percepções de competência física das crianças. $\mathrm{O}$ consentimento dos pais e/ou responsáveis foi obtido para cada uma das crianças envolvidas neste estudo.

\section{Medidas:}

Percepções de Competência. A subescala da parte fisica da Pictorial Scale of Perceived Competence and Social Acceptance (PSPCSA) de Harter e Pike (1984) para crianças foi utilizada para medir percepções de competência física. Esta subescala consiste de seis itens (balançar, subir, amarrar sapatos, saltitar, correr e saltar com 1 pé). Elas são apresentadas em figuras, cada página contém duas figuras colocadas uma ao lado da outra; uma figura representa uma criança que é competente e a outra representa uma criança que não é tão competente. A criança deverá primeiro selecionar a figura mais parecida com ela e então decidir o quanto ela é parecida com a criança da figura. A amplitude de resultados para cada item em cada subescala é de 1 (baixa competência) a 4 (alta competência). A fidedignidade para consistência interna e a validade da subescala física têm sido demonstradas em vários estudos (WHSS, 1991,1995).

Desempenho Motor. O teste TGMD (ULRICH, 1985) foi usado para acessar a competência nas habilidades motoras de cada criança envolvida no presente estudo. O TGMD é um teste usado para avaliar desempenho motor amplo e inclui 12 itens dos quais 7 são habilidades de locomoção (correr, galopar, saltitar, salto sobre o mesmo pé, salto como os dois pés, salto com 1 pé, e corrida lateral) e 5 são habilidades de controle de objeto (rebater, quicar, receber, chutar, arremessar). O TGMD é um teste múltiplo que obtém informação sobre a capacidade nas habilidades de locomoção e controle de objetos de indivíduos. O TGMD permite uma avaliação 
separada de cada subescala (locomoção e controle de objeto). Entretanto, o teste não permite a avaliação separada de cada habilidade motora uma vez que elas estão integradas no modelo estatístico que valida o teste. A aplicação do teste leva aproximadamente 20 minutos por criança. O protocolo de aplicação sugere o uso de câmara filmadora para registro e posterior análise do desempenho motor. A análise do vídeo tape leva aproximadamente 30 minutos por criança. Os escores relatados no teste incluem dados brutos, padrão e percentil para cada uma das duas subescalas do TGMD (habilidades de locomoção e de controle de objeto). Considerando os dados brutos, o resultado mais baixo para cada subescala é zero e o mais alto é de 26 para a subescala das habilidades de locomoção e 19 para subescala das habilidades de controle de objeto, com um somatório total de 45 pontos no máximo. Os dados brutos são obtidos através do somatório de pontos recebidos pelo indivíduo na execução de cada habilidade motora, considerando-se a forma do movimento executado pelo indivíduo em cada tentativa $O$ percentil também é fornecido e tem sido constantemente usado como medida complementar no entendimento dos resultados do teste. No presente estudo dois examinadores foram responsáveis pela avaliação das habilidades motoras do $T G M D$, o coeficiente de consistência entre estes avaliadores foi de 0,90 , similar ao coeficiente obtido pelo autor do teste.

\section{Procedimento:}

Cada criança foi filmada enquanto executava o teste de habilidades motoras (TGMD) em suas respectivas escolas. O protocolo padronizado sugerido pelo autor do teste (ULRICH, 1985) foi usado na administração do mesmo. Ao término do TGMD as crianças responderam individualmente as questões referentes às percepção de competência (PSPCSA), seguindo o protocolo estabelecido para esta avaliação.

\section{Resultados}

\section{Percepção de Competência:}

Duas One WayANOVA (análises de variância) foram utilizadas para investigar a influência da idade e do gênero nas percepções de competência física de crianças. Os resultados evidenciam que não foram encontradas diferenças significativas em percepções de competência física com relação a idade, $\mathrm{F}(2,81)=0,35 ; \mathrm{p}=0,70$, e ao gênero, $F(1,82)=0,12 ; p=0,73$. Conforme as hipóteses estabelecidas neste estudo, meninos e meninas evidenciam percepções similares, bem como grupos de crianças de diferentes idades (Referir-se a Tabela 1 para as médias dos grupos de idade e gênero em relação a percepção de competência física).

\section{Desenvolvimento Motor:}

Uma análise multivariada foi utilizada para avaliar os efeitos do fator gênero e idade no desenvolvimento motor, utilizando como critério Wilks'Lambda (L). Resultados evidenciam diferenças significativas para grupos de idade, $\mathrm{A}=0,76, \mathrm{~F}(2,83)=6,00, \mathrm{p}_{-}=.000$, e gênero, $A=0,81, F(2,83)=9,74, p=.000$.

Habilidades de Locomoção: Os resultados da multivariada evidenciaram diferenças significativas nas habilidades de locomoção em relação as diferentes idades, $\mathrm{F}(2,86)=13,01, \mathrm{p}=0,001$. Testes estatísticos de continuidade, três pares de testes-t independentes, foram aplicados para avaliar o desenvolvimento locomotor diferenciado nos grupos etários. Os testes de continuidade demonstram que crianças mais velhas tendem a exibir desempenho locomotor superior comparado ao desempenho dos mais jovens. Crianças entre 9 e 10, evidenciam desempenho locomotor significantemente superior quando comparadas ao grupo de 7 e 8 anos, $t$ (66) $=1,97, \mathrm{p}=0,05$, e ao grupo de 5 e 6 anos, $\mathrm{t}(32)=$ $3,68, \mathrm{p}=0,00$. Anda, crianças de 6 e 7 anos evidenciam desempenho locomotor significativamente superior quando comparadas ao grupo de crianças de 5 e 6 anos, $\mathrm{t}(72)=4,14, \mathrm{p}_{-}=0,0001$ (Tabela 1). Estes resultados suportam, em parte, a hipótese deste estudo de que crianças mais velhas exibem um desenvolvimento locomotor mais qualificado quando comparadas as mais jovens.

Com relação ao gênero, não foram encontradas, diferenças na habilidade de locomoção, $\underline{\mathrm{F}}(1,87)=0,17$, p $=0,67$. Meninos e meninas evidenciaram desempenhos similares, conforme hipóteses estabelecidas. 
Table 1

Percepções de Competência Física e Desempenho Motor

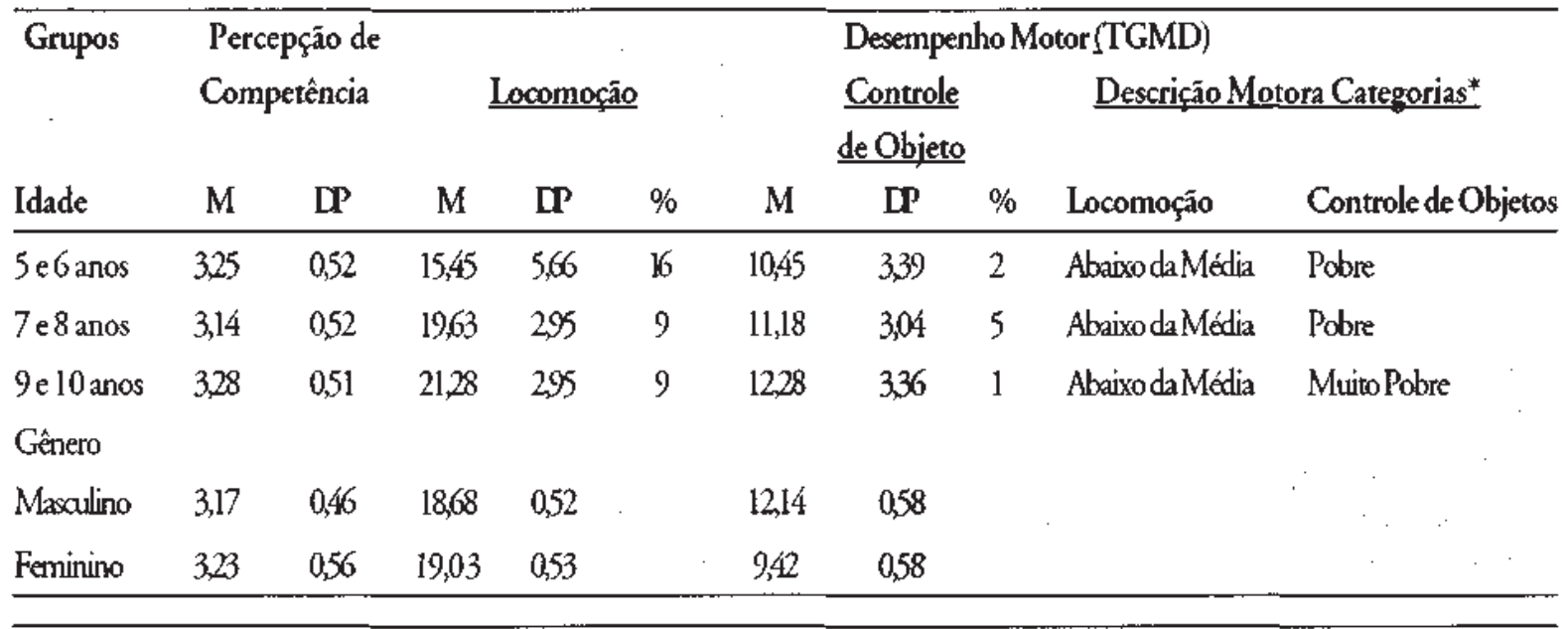

Nota. Sete categorias de desempenho motor são previstas pelo autor do teste TGMD (Ulrich, 1985) estruturadas a partir dos dados brutos: Muito Pobre, Pobre, Abaixo da Média, Média, Acima da Média, Superior e Muito Superior.

Habilidades de Controle de Objetos: Os resultados da análise multivariada não evidenciaram diferenças significativas nas habilidades de controle de objetos em relação as diferentes idades, $F(2,86)=1,00, p=0,37$. Crianças dos diferentes grupos etários evidenciam desempenhos similares. Estes resultados contrariam, em parte, a hipótese proposta no presente estudo.

Com relação ao gênero, confirmando a hipótese deste estudo, foram encontradas diferenças significativas nas habilidades de controle de objetos, $\mathrm{F}(1,87)=17,02, \mathrm{p}$ $=0,000$. Meninos evidenciam desempenhos significantemente superiores as meninas na habilidade de controle de objetos.

Relações entre Percepção de Competência e Desenvolvimento Motor

Uma análise de regressão múltipla foi conduzida para avaliar as relações entre desenvolvimento motor atual e percepções de competência, mais especificamente, se desenvolvimento motor atual é um preditor de percepção de competência física, e se fatores como idade e gênero alteram estas relações. O método enterwise foi utilizando usando percepção de competência como variável dependente e desenvolvimento motor total como variável independente. Desenvolvimento motor atual não foi um preditor significante de percepção de competência, com uma combinação linear entre percepção de competência e desenvolvimento motor atual fraca $\left(R=0,22, R^{2}=0,05\right)$ e não significante $(F=2,20$, $\mathrm{p}=0,14)$. Ou seja, somente $5 \%$ da variabilidade nas percepções de competência foram preditas pelo desenvolvimento motor atual. Adicionando ao modelo de regressão múltipla idade $(\mathrm{r}=+0,23, \mathrm{p}=0,31)$ e gênero $(\mathrm{r}=+0,23, \mathrm{p}=0,51)$ não levou ao fortalecimento e ou significância da mesma. Confirmando as hipóteses deste estudo, de que meninos e meninas de diferentes idades não são precisos em suas percepções de competências individuais e que desenvolvimento motor atual não se configura como preditor de percepção de competência.

\section{Discussão}

Este estudo foi conduzido com o objetivo de investigar as percepções de competência e níveis de desenvolvimento motor de crianças em relação a diferentes idades e ao gênero, bem como as relações entre 
percepção de competência física e desenvolvimento motor atual de meninos e meninas de diferentes idades.

\section{Percepção de Competência:}

Os resultados sugerem que crianças de diferentes idades e gênero evidenciam percepções de competência similares. Em relação a idade, ficou evidenciado um platô nas percepções de competência. Congruente com o estudo conduzido por Urich (1987), as crianças entre 5 e 10 anos investigadas no presente estudo evidenciaram médias relativamente altas (aproximadamente 3,2) permanecendo alta nos diferentes grupos etários. Este platô pode ser explicado pela falta de parâmetros motores da criança para julgar suas competências. Algumas pesquisas (HARTER, 1982;NICHOLLS, 1984) têm demonstrado decréscimos dramáticos em percepções de competência de crianças a partir de 8 anos de idade em decorrência de uma conscientização de potencialidades e ou capacidades pessoais. Para que esta conscientização ocorra, se faz necessário o estabelecimento de pré-requisitos que permitam a esta criança observar, praticar, receber feedback, aprender e estabelecer parâmetros para o seu desempenho, o que não se configura na realidade da prática motora das crianças aqui investigadas. Nos preocupa a superestimação de um desempenho motor pobre, principalmente nas crianças mais velhas. Superestimar suas competências pode levar estas crianças a constantes fracassos frente à tarefas que thes parecem fáceis, porém em realidade se configuram como acima de suas possibilidades motoras, influenciando negativamente a motivação para atividades físicas e esportivas futuras.

Em relação as percepções de competência de meninos e meninas, os resultados deste estudo estão em concordância com alguns estudos prévios os quais observaram semelhanças nas percepções de competêncknosgêneros(ANDERSON, 1985;GOODWAY, 1996, 1997;HANSFORD, 1982;VALENT1NI, 1999;WYLIE, 1979). Este dado, nos parece importante na medida em que meninos e meninas parecem estar construindo um sistema de percepção no domínio físico que não é diferenciado pelo gênero. Este tópico têm se evidenciado contraditório na literatura. Investigações mais detalhadas se fazem necessárias para contribuir no discernimento das variáveis que interferem em construções de percepções de competência similares e ou diferenciadas entre gêneros. Fatores como idade, parâmetros prévios, nível de desempenho, tipos diferenciados de prática, contexto familiar e social, entre outros, talvez exerçam alguma forma de influência nas percepções de competência de crianças.

\section{Desenvolvimento Motor}

Em relação ao desenvolvimento motor os resultados sugerem um desempenho em geral abaixo da média e pobre. Em relação as comparações dos grupos etários, os resultados mostram que crianças mais velhas apresentam desempenho superior quando comparadas as crianças mais jovens nas habilidades de locomoção. Entretanto com relação as habilidades de controle de objeto, contrariando nossa expectativa, não foi observada uma superioridade significativa no desempenho motor de crianças mais velhas ao serem comparadas com as mais jovens. A expectativa de que crianças mais velhas evidenciem habilidades motoras mais qualificadas reside na experiência e prática destas habilidades no decorrer dos anos. As habilidades motoras não emergem naturalmente, elas são a conseqüência de oportunidades para experienciar atividades motoras apropriadas e sistemáticas. O desenvolvimento motor das crianças sugere a falta de oportunidades da prática de habilidades motoras em que se manipulam objetos, bem como a carência de instrução nestas habilidades. Somente a prática e a instrução podem levar crianças, no decorrer dos anos escolares, a níveis mais sofisticados de habilidades motoras.

Em relação ao gênero, meninos e meninas demonstraram desenvolvimentos similares nas habilidades de locomoção e diferenças nas habilidades de controle de objeto, sugerindo um desempenho mais eficiente a favor dos meninos nas habilidades de chutar, quicar, arremessar, rebater e receber. Esta mesma tendência é observada em vários estudos prévios (DINUCCI, 1976;GOODWAY, 1997;MORRIS, 1982; ULRICH， 1987;VALENTINI， 1999). Meninos parecem 
ser mais motivados, na sociedade atual, a envolver-se e praticar com maestria atividades de controle de objetos. Os meios de comunicação, o mercado de brinquedos e as próprias famílias tendem a considerar estas habilidades como mais relacionadas ao sexo masculino. Este dado nos lança o desafio de propiciar oportunidades iguais para meninos e meninas experienciarem atividades motoras que envolvam o controle e manipulação de objetos nas aulas de educação física e/ou programas esportivos.

\section{Relações entre Percepção de Competência e De- senvolvimento Motor}

A extensão com que percepções de competência e desenvolvimento motor atual estavam relacionadas foi avaliado no presente estudo. A expectativa de que crianças em geral são pouco precisas na percepção de seus desempenho motores atuais, e que esta relação não tende a ser fortalecida pelo aumento da idade e pelo gênero, foi confirmada.

Estudos prévios sugerem que com o desenvolvimento as crianças se tornam cognitivamente mais capazes de realizarem uma auto-avaliação realística de suas competências afastando-se da superestimação observada quando mais jovens, especialmente entre 5 e 6 anos de idade (OVERBY,1994). Entretanto, tornar-se cognitivamente mais capaz de auto- avaliar-se não é um fator que ocorre por si conforme a criança se desenvolve, ele é sim construído através das experiências de movimento adequadas que estas crianças vivenciam. $\mathrm{Ou}$ seja, através da instrução e prática mediada pela interação com outros.

Portanto, na perspectiva teórica adotada neste estudo, as explicações do porque crianças não são precisas sobre seus desempenhos motores atuais leva em consideração os quatro fatores propostos por Harter (1978), ou seja, experiências passadas, dificuldades e desafios associados com o produto/tarefa, interação e feedback de outras pessoas e a motivação intrínseca. As crianças que tomaram parte deste estudo demonstraram em geral um desempenho motor abaixo da média nas habilidades locomotoras e pobre nas habilidades de con- trole de objetos, de acordo com o sistema avaliativo proposto no teste adotado (ULRICH, 1985). O desempenho motor desta crianças é caracterizado pela falta de experiências e dificuldades de evidenciar padrões de movimento mais avançados ou qualificados, mesmo com o desenvolvimento. Os resultados sugerem que a falta de experiência e a conseqüente carência de parâmetros motores interfere na precisão com que estas crianças julgam seus desempenhos.

O terceiro e quarto fatores propostos por Harter (1978), suporte e interação com outras pessoas que são importantes para a esta criança e motivação intrínseca, parecem também influenciar estes resultados na medida em que estas possuem poucas oportunidades de prática apropriada. Observa-se uma carência de oportunidades de prática da educação física sistemática, com o apropriado feedback e suporte de professores, bem como poucas possibilidades de interação e trocas com outras crianças. A pratica da educação física em todas as escolas que fizeram parte do estudo parece ser conduzida de forma recreativa opcional, sem uma pratica sistemática de habilidades motoras fundamentais. As habilidades motoras fundamentais, como já discutimos anteriormente, não emergem naturalmente, elas se constróem a partir de oportunidades de experiências em atividades de movimento, da atenção individualizada, da mediação do feedback constante e específico, e da instrução apropriada baseada nas necessidades de todas as crianças. Estes fatores são determinantes para ajudar a crianças a melhor conhecerem suas competências e a tornarem-se mais competentes de fato. Perceber-se competente na execução de diferentes tarefas motoras conduz ao aumento da motivação intrínseca para a conquista em atividades físicas e esportes (HARTER,1982;NICHOILLS, 1984).

\section{Conclusões e Repercussões para a Prática da Educação Física}

A literatura sugere que discrepâncias entre percepções de competência e desenvolvimento motor real influenciam negativamente a motivação da criança para persistir naatividadefisica(GOODWAY, 1997;STIPEK, 1990; RUDISILL, 1993;ULRICH, 1987;WEISS, 1990).Éimpor- 
tante propiciar para crianças oportunidades para engajar-se em atividades motoras que promovam o desenvolvimento motor e as percepções de competência fisica; particularmente para crianças que evidenciam um desempenho motor pobre. Portanto, percepções de competência precisas são críticas para que as crianças continuem engajadas em atividades que incorporam as habilidades motoras fundamentais. Os resultados deste estudo sugerem que professores de educação física precisam desenvolver programas que não somente tenham como alvo as melhorias de desenvolvimento motor, mas também as percepções de competência das crianças para que as mesmas se tornem mais precisas ao estimar seus desempenhos motores. Para que a instrução motora se torne efetiva, nesta perspectiva, é fundamental: (1) propiciar experiências motoras prazerosas, assegurando-se que as crianças possuem oportunidades otimizadas e suficientes para a prática; (2) propiciar estratégias instrucionais eficientes e variadas baseadas na avaliação da habilidade motora e do desenvolvimento motor do aluno; (3) identificar os componentes críticos de cada habilidade motora para assegurar que os mesmos são enfatizados via demonstração, descrição verbal, cartazes, entre outras estratégias de aprendizagem; (4) encorajar a criança a refletir sobre seu desempenho; (5) propiciar a experiência de tarefas com diferentes graus de dificuldade e permitir as crianças possam escolher o nível de dificuldade na tarefa que mais lhe desafie, (6) propiciar, durante a instrução, feedback pessoal e de grupo, positivo e significativo relacionado com o desenvolvimento das habilidades motoras; (7) construir estratégias que promovam motivação intrínseca; (8) elogiar conquistas reais que resultaram do esforço na aprendizagem.

O presente estudo indica que crianças evidenciam níveis pobres de desenvolvimento motor e percepções de competências elevadas, ou seja não são precisas em suas percepções. Quando oportunidades são propiciadas para conhecer, experienciar e aprender habilidades motoras como resultado de uma instrução apropriada, sistemática e significativa para cada criança as possibilidades de ganhos motores são otimizados e a as percepções de competência se tornam mais precisas. Considerando a importância das percepções de competência na motivação da criança para as conquistas em atividades físicas, e esportivas é necessário propiciar a todos, meninos e meninas de diferentes idades, com instrução e oportunidades de movimento planejadas para desenvolver competências motoras e percepções de competência positivas e realistas.

\section{Referências}

ANDERSON, P. L; ADAMS, RJ. The relationship of fiveyear-olds'Academic readiness and perceptions of competence and acceptance. Journal of Pediatrics, 116, p. 955-959, 1985 .

DiNUCCI,J\&MGROSS.Motor performance: a comprehensive analysis of age and sex differences between boys and girls ages 6 to 9 years. In: BROEKHOFF, S. (Ed.). Physical education, sports andthe sciences. Eugene, OR: Microform Pub., UniversityofOregon. p. 108-127.

FELTZ \& BROWN, Perceived competence in soccer skills among young soccer players. Journal of Sport Psychology, 6,385-394.1984.

FERRAZ, O.L Desenvolvimento do padrão fundamental de movimento correr em crianças: um estudo semilongitudinal. Revista Paulista de Educação Física,6 (1), 2634,1992 .

GABBARD, C. P. Lifelong motor development. 3. ed. Madison Dubuque, IA: Brown \& Benchmark, 2000.

GARCIA, C. GARCIA, L.Examining Developmental changes in Throwing. In Clark, J.E. e Humphrey, J.(Eds) Motor Development: Research and Reviews. 2002.

GOODWAY-SFUEBLER,J.D. The effect of motor skill intervention on the fundamental motor skills and susteined activity of African-american preescholers who are at-risk. Unpublished doctoral dissertation, Michigan State University,EastLansing. 1994.

GOODWAY,J.D.,RUDISILL,M.E., VALENTINI,N.C. The influence of instruction on the development of catching in young children. In Clark, J.E. e Humphrey, J.(Eds) Motor Development: Research and Reviews. 2002.

GOODWAY, J. D.; RUDISILL,M. E. Influence of a motor skill 
intervention program on perceived competence of at-risk African American preschoolers. AdaptedPhysicalActivity Qmrterly, 13, p. 288-2301,1996.

GOODWAYJ.D.;RUDISIII,M.EPerceivedphysical competence and actual motor skill competence of African American preschool children. AdaptedPhysicalActivity Qmrterly, 14, p. 314-326,1997.

HAMILTON,M.L;TATE,A.Constraints of throwing behavior of children. In Clark, J.E. e Humphrey, J.(Eds) Motor Development: Research and Reviews. 2002.

HAMILTON,ME;GOODWAY,J.D.;HAUBENSTRICK ER,J. Parent-assisted instruction in a motor skill program for at-risk preschool children. AdaptedPhysicalActivity Qmrterly, 16 (4), 415-426,1999.

HANSFORD,B.C.;HATITE,J.AThe relationship between self and achievement / performance measures. Review of Educational Research, 52, p. 123-142,1982.

HARTER S. Effectance motivation reconsiderei toward a developmental model. Human Development, 21, p. 34-64, 1978.

HARTER, S. The perceived competence scale for children. Child Development, 53, p. 87-97,1982.

HARTER S.; PIKE, R The pictorial scale of perceived competence and social acceptance for young children. Child Development, 55, p. 1969-1982,1984.

HARTER,S.WHITESELL,N.R.;KOWALSKI,P. Indi

vidual differences in the effects of educational transitions on young adolescente perceptions of competence and motivational orientation. American Educational Research Journal,29, p.777-807,1992.

HAYWOOD,K.M.; GETCHELL,N. Lifespan motor development. 3. ed. Champain, IL: Human Kinetics, 2001.

KLINT,KA;WEISS,M.R. Perceived competence and motives for participating in youth sports: a test of Harter's competencemotivation theory. Sport Psychology, 9,55-56. 1987

\section{LANGERDORFEN,S.ROBERTON,A.M.,Developrnent} profiles in overarm throwing: searching for attractors, stages, and constrainst. In Clark, J.E. e Humphrey, J.(Eds) Motor Development: Research and Reviews. 2002.
MENDES,EG.O.;GOBBI,LT.B.ODesempenho do padião fundamental domovimento de receber em crianças de 5 a 8 anos, em função da idade e sexo. In: BENTO, J., MARQUES, A. As ciências do desporte e a prática desportiva. V. 1 .Porto: Universidade do Porto, Faculdade de Ciências do Desporto e Educação Física. 1991.

MORRIS,A.;WILLIAMS,

J.;ATWATER;WILMORE,J.Age and sex differences in motor performance of 3 through 6 year old children.

Research Quarterlyfor Exercise and Sport, 55, p. 288297, 1982.

NEWELL, K. Physical constraits to development of motor skills.In J.THOMAS(Ed),Motor development during prescholl and elementary years (pp 105-120). Minneapolis: Burgess. 1984.

NICHOLLS, J. G. Conceptions of ability and achievement motivadon. In: AMES, R; AMES, C. (Ed.). Research on motivation in education: v. 1. New York: Academic Press, 1984, p. 39-73.

\section{OVERBY,BRANTA,GOODWAY,\&SMITH. The} relashionship of parental attitudes to perceived competence, motor development, and physical fitness of at risk youth. Paper presented at the national Convention of the American AUience for Health, Physical Education, Recreation, and Dance, Denver, CO, 1994.

PAYNE, V. G.; ISAACS, L D. Human motor development: a lifespan approach. 4. ed. Mountain View, CA: Mayfield, 1999.

PELLEGRINI,A.M.;CATUZZO,M.T.Estágiosnaaquisição do padrão motor de correr: avaliação em escolares brasileiros. In: BENTO, J.,MARQUES,A As ciências do desporte e a prática desportiva. V. 1 .Porto: Universidade do Porto, Faculdade de Ciências do Desporto e Educação Física. 1991.

RUDISILL, M. E. Influence of perceived competence and causai dimension orientations on expectations, persistence and performance during perceived failure. Research Quarterlyfor Exercise andSport, 60,166-175.1989 RUDISILLME;MAHAR,M.T;MEANEY,KS.The relationship between childrens perceived and actual motor competence. Perceptual and Motor Skills, 76, p. 895-906, 1993.

RUDISILL,M.E;PEMBERTON,C.L. Sex differences on 
various cognitive parameters under conditions of success and failure for three motor tasks. Psychology and Sociology of Sport, v.2,p. 3343,1990.

SEEFELDT, V.; HAUBENSTRICKER,J. Pattems, phases, or stages: an analytical model for the study of developmental movement In:KELSO,J.A;CLARK,J.E.(Ed.). The development ofmovement control and coordination. New York: Wiley, 1982.

SOUTHARD, D. Control Paremeters for the Development of Throwing. In Clark, J.E. e Humphrey, J.(Eds) Motor Development: Research and Reviews. 2002.

STIPEK,D.J.;DANIELS,D.H. Children's use of dispositional attributions in predicting the performance behavior of classmates. Journal of Applied Development Psychology, 11, 13-28.1990.

STIPEK, D. J.;KOWALSKI, P. S. Learned helplessness in taskoriented versus performance-oriented testing conditions. Journalof Educational Psychohgy, 81, p. 384-391,1989.

SURDI, AC.,KREBS,RJ.Estudos dos padrões de movimento de pré-escolares que participaram do programa de desenvolvimento infantil do SESI da cidade de Videira SCKLNESIS.21,57-69.1999.

ULRICH, D. The testofgross motor development. Austin, TX:Prod-Ed, 1985.

ULRICH, B. D. Perceptions ofphysical competence, motor competence, and participation in organized sport: Their interrelationships in young children. Research Quarterly for Exercise and Sport, 58, p. 57-67,1987.

VAI^NIININ.QRUDMLJvIE\&COODWAY,JD. Masteiy climate: Children in charge of their own learning. TeachingElementary Physical Education. 1999.

WEISS,M.R;HORN,T.S.The relation between children's accuracy estimates of their physical competence and achievement related characteristics. Research Quarterly for ExerciseandSport, 61,250-258.1990

WEISS, M. R. Psychological skill development in children. The Sport Psychologist, 5, p. 335-354,1991.

WEISS, M. R Children in sport: An educational model. In: MURPHY, S. M. (Ed.), Sport Psychology Interventions. Champaign, IL: Human Kinerics, 1995. p. 36-69.
WYLIE, R. The self-concept: theory and research on selected topics. v. 2.

Lincoln: UniversityofNebraska Press, 1979.

\section{Notas}

'Universidade Federal do Rio Grande do Sul

Escola de Educação Física

Autor Correspondente: Nadia Cristina Valentini, PhD.

Comportamento Motor

E-mail: nadiacv@esef.ufrgs.br

Enviado em: 16/06/02

Revisado em:

16/07/02 Aceito em:

$24 / 07 / 02$ 